\title{
Dependency of endothelial cell function on vascular smooth muscle cells in guinea-pig mesenteric arteries and arterioles
}

\author{
Yoshimichi YAMAMOTO ${ }^{1,2}$ and Hikaru SUZUKI ${ }^{2}$ \\ ${ }^{1}$ Laboratory of Physiology, Nagoya City University School of Nursing, \\ ${ }^{2}$ Department of Regulatory Cell Physiology, Nagoya City University Graduate School of \\ Medical Sciences, Mizuho-Ku, Nagoya 467-8601, Japan
}

\begin{abstract}
Using guinea-pig mesenteric arteries and arterioles, we investigated the membrane potential of endothelial cells at rest and during application of acetylcholine (ACh) with and without the smooth muscle layers attached. When smooth muscle and endothelial layers were in close apposition, the resting membrane potentials of the two types of cells were closely related and were slightly more negative in the smooth muscle cells than in the endothelial cells. Once the endothelial layer was separated from the smooth muscle layer, the endothelial cells depolarized (the average, $-4.2 \mathrm{mV}$ ). In the isolated endothelial layer, ACh did not induce a membrane hyperpolarization as expected, but did induce a quick depolarization soon after conventional whole-cell recording was started. However, as the pipette solution (high $\mathrm{K}^{+}$) gradually diffused into the endothelial layer, the membrane response to ACh gradually changed toward hyperpolarization. ACh-induced hyperpolarization was also observed after incubating preparations in a high-potassium bath solution. Our results indicate that vascular smooth muscle cells and endothelial cells are influencing each other as a functional unit and that the endothelial cells rely on the smooth muscle cells for their intracellular ionic composition and resting membrane potential.
\end{abstract}

Key words: endothelial cell, gap junction, smooth muscle, membrane potential, $\mathrm{EDHF}$

\section{Introduction}

It is now well established that vascular endothelial cells produce vasoactive substances such as nitric oxide and prostacyclin, and control vascular smooth muscle contractility. They may also do so by affecting the membrane potential of smooth muscle cells via myoendothelial gap junctions (Yamamoto et al., 1999). On the other hand, it is not clear whether smooth muscle cells control endothelial cell function or not. A vascular endothelium consists of a mono-cell layer of endothelial cells which are thin and flat, with an intracellular capacity which seems to be much

Correspondence to: Yoshimichi Yamamoto, Ph.D., Professor, Department of Regulatory Cell Physiology,

Nagoya City University Graduate School of Medical Sciences, Mizuho-Ku, Nagoya 467-8601, Japan

Phone: +81-52-853-8131, 8863 Fax: +81-52-842-1538 e-mail: yyamamot@med.nagoya-cu.ac.jp 
smaller than that of the smooth muscle cell layer. As there are ionic passages between the two layers via myoendothelial gap junctions, it is very likely that the intracellular ionic composition in endothelial cells is influenced by the much larger capacity of the smooth muscle cells.

Myoendothelial gap junctions are heterocellular couplings between smooth muscle and endothelial cells. As these couplings do not exhibit rectification (Yamamoto et al., 2001), ionic current can flow through them in either direction, driven by the potential difference between the two types of cells. Thus, acetylcholine (ACh)-induced hyperpolarizations are conducted from endothelial cells to smooth muscle cells and $\mathrm{Ba}^{2+}$-induced action potentials are conducted in the opposite direction (Yamamoto et al., 2001). Heterocellular gap junctions have also been studied between cultured neurons and glia cells taken from the rat locus ceruleus (Alvarez-Maubecin $e t$ $a l ., 2000)$. These authors suggested that the glia with their negative membrane potential ( -80 $\mathrm{mV}$ ) would provide a steady hyperpolarizing current into neurons and modulate their firing frequency, thus facilitating synchronization of spontaneous activity.

In the present experiments, we used guinea-pig mesenteric arteries and arterioles to investigate the membrane potential of endothelial cells at rest and during application of ACh with and without the smooth muscle layers attached. Our results indicate that vascular smooth muscle cells and endothelial cells are influencing each other as a functional unit and that the endothelial cells rely on the smooth muscle cells for their intracellular ionic composition and resting membrane potential.

\section{Methods}

\section{Preparations}

Male guinea-pigs, weighing 200-300 g, were anaesthetized by sevoflurane inhalation and killed with an animal guillotine. Animals were treated ethically according to the rules of the Laboratory Animal Care and Use Committee of Nagoya City University Graduate School of Medical Sciences. From the mesenteric vasculature of the ileal region, first-order mesenteric arterioles (diameter; $50-100 \mu \mathrm{m}$ ) and second or third branches of mesenteric arteries (diameter; $300-400 \mu \mathrm{m}$ ) were dissected out. The arterioles were incubated in collagenase solution (nominally $\mathrm{Ca}^{2+}$-free physiological saline containing $0.5 \mathrm{mg} \mathrm{ml}^{-1}$ collagenase; Wako Pure Chemical Industries, Ltd., Osaka, Japan) for $15 \mathrm{~min}$ at $37^{\circ} \mathrm{C}$, and then the adventitial layer removed mechanically to expose the smooth muscle layer. The endothelial layer was also exposed by cutting a part of the preparation open and simultaneous recordings made from both smooth muscle and endothelial cells.

Isolated endothelial layers were prepared from mesenteric arteries in two ways. In abluminal-surface-up preparations, a segment of artery was everted and incubated in collagenase solution for $50 \mathrm{~min}$ at $37^{\circ} \mathrm{C}$, after which the everted artery was pressed down onto a glass cover slip. This procedure transferred the endothelial layer alone onto the cover slip with the abluminal surface upwards, allowing individual cells to be clearly distinguished with a phasecontrast microscope (Fig. 1A). In luminal-surface-up preparations, a segment of artery was incubated in collagenase solution for $30-40 \mathrm{~min}$ at $37^{\circ} \mathrm{C}$, and then the adventitial and smooth muscle layers were removed mechanically leaving the endothelial layer surrounded by the 
A

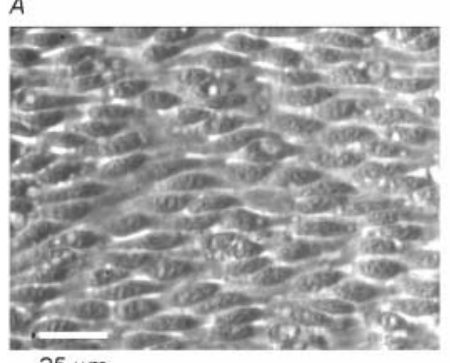

$25 \mu \mathrm{m}$

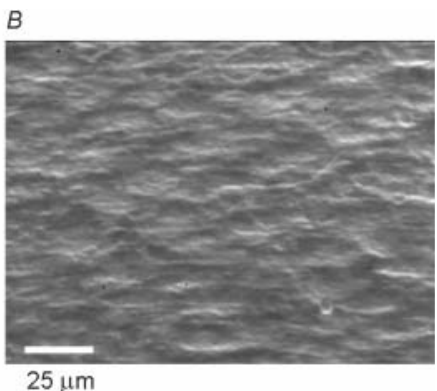

$25 \mu \mathrm{m}$

Fig. 1. Phase-contrast micrographs of isolated endothelial layers in mesenteric artery preparations. A: An abluminal-surface-up preparation containing only endothelial cells, in which individual cells can be clearly distinguished. $B$ : A luminal-surface-up preparation where the individual endothelial cells are obscured by the internal elastic lamina.

internal elastic lamina. The isolated endothelial layer was cut open and placed with the luminal surface upward. Because of the internal elastic lamina, individual cells could not be clearly distinguished with phase-contrast microscopy (Fig. $1 B$ ). The preparation was fixed in a culture dish and superfused with preheated $\left(30^{\circ} \mathrm{C}\right)$ and aerated $\left(100 \% \mathrm{O}_{2}\right)$ bath solution at a constant rate $\left(1 \mathrm{ml} \mathrm{min}^{-1}\right)$.

\section{Electrophysiological techniques}

Membrane potentials of the smooth muscle and the endothelial cells were measured in the current clamp mode of the conventional whole-cell clamp method. Voltage signals were acquired using either a combination of EPC-7 (List-Medical, Darmstadt, Germany) and Axopatch 200 (Axon Instruments, Foster City, CA, U.S.A.) systems or a MultiClamp 700A system (Axon Instruments). These signals were digitized with either a Digidata $1200 \mathrm{~B}$ or a Digidata 1322A data acquisition system (Axon Instruments). The sampling rate was always fivetimes higher than the cut-off frequency $(-3 \mathrm{~dB})$ of a low-pass Bessel filter. Current clamp protocols were controlled with pCLAMP 8 or 9 software (Axon Instruments). Numerical data are represented as the mean \pm standard error.

\section{Solutions and chemicals}

The composition of the standard bath solution was (mM): $\mathrm{NaCl}, 141.5 ; \mathrm{KCl}, 5.4 ; \mathrm{CaCl}_{2}, 1.8$; $\mathrm{MgCl}_{2}, 1$; Hepes, 10; glucose, 5. The $\mathrm{pH}$ was adjusted to 7.3 using $\mathrm{NaOH}$. The composition of the high-potassium bath solution was (mM): $\mathrm{KCl}, 143 ; \mathrm{MgCl}_{2}, 1$; Hepes, 10; glucose, 5 . The $\mathrm{pH}$ was adjusted to $7.3 \mathrm{using} \mathrm{KOH}$. The pipette contained a solution of (mM): $\mathrm{KCl}, 143 ; \mathrm{MgCl}_{2}, 1$; EGTA, 5; Hepes, 10; glucose, 5. The $\mathrm{pH}$ was adjusted to 7.3 using $\mathrm{KOH}$. ACh was obtained from Sigma (St. Louis, MO, U.S.A.).

\section{Results}

In guinea-pig mesenteric arterioles, the resting membrane potentials of smooth muscle and 

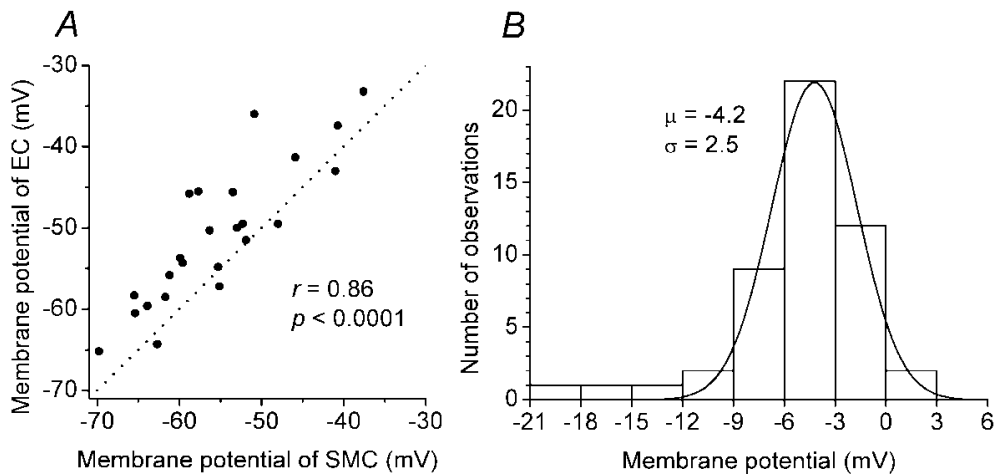

Fig. 2. A: Relationship of the resting membrane potentials between smooth muscle cells (SMC) and endothelial cells (EC). Membrane potentials of the two types of cells were recorded simultaneously in 24 intact mesenteric arterioles. Each point represents the data from one arteriole. The dotted line represents $y=x$. $B$ : Distribution of the resting membrane potential of endothelial cells measured from 50 isolated endothelial layers from mesenteric arteries (22 abluminal-surface-up and 28 luminal-surface-up preparations). The bin width was set to $3 \mathrm{mV}$ and the number of observations within each bin was displayed as the height of column. The continuous curve represents a Gaussian function which can fit most part of the distribution except for the three outliers.

endothelial cells were measured simultaneously soon after conventional whole-cell recording was established in the current-clamp mode (Fig. $2 A$ ). The resting membrane potentials of the two types of cells were closely related, with a correlation coefficient of $0.86(P<0.0001)$. Regression analysis was not performed because both variables were expected to have a similar degree of error, but instead the data were compared with the $y=x$ line (dotted line in Fig. 2A). Most of the data points fell above the line of $y=x$, indicating that the measured resting membrane potentials were more negative in smooth muscle cells than in endothelial cells. While four points fell closely below the $y=x$ line, if some degree of error in the voltage measurements were taken into account, it could be concluded that the resting membrane potentials were slightly more negative in smooth muscle cells than in endothelial cells.

In mesenteric arteries, once the endothelial layer was separated from the underlying smooth muscle layer, the resting membrane potential of the endothelial cells was much less negative than that measured in the preparations having the smooth muscle layers attached (Fig. $2 B$ ). The histogram shows the distribution of the resting membrane potentials measured in both luminal-surface-up and abluminal-surface-up preparations made from mesenteric arteries. As the membrane potentials in the two types of preparation were similar, all data were combined. Excluding the three outliers with relatively more negative values, the distribution fitted a Gaussian function with an average of $-4.2 \mathrm{mV}$.

As ACh produces membrane hyperpolarization in isolated endothelial cells (Busse et al., 1988; Sakai, 1990), the membrane potential response to ACh was examined in isolated endothelial cell layers of mesenteric arteries (Fig. 3). Soon after conventional whole-cell recording was started in the current-clamp mode, ACh did not induce a membrane hyperpolarization, but instead induced a quick depolarization. If this depolarization were also produced by potassium 


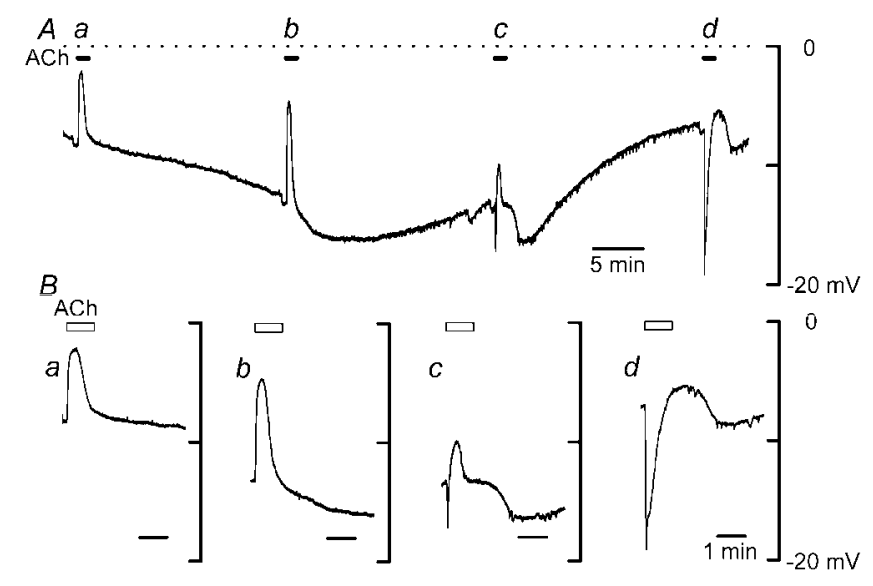

Fig. 3. The time-dependent change in the ACh-induced voltage response in a luminal-surfaceup mesenteric artery preparation. A: The membrane potential of an endothelial cell within the isolated endothelial layer was recorded. ACh $(3 \mu \mathrm{M})$ was applied during the periods indicated by the four bars. Small negative voltage deflections prior to the ACh-responses were artefacts on switching the bath solution. $B$ : ACh-induced voltage responses in expanded time scale. $a-d$ correspond to $a-d$ in $A$. ACh was applied during the periods indicated by the open boxes. Scales given in $d$ also apply to $a-c$.

channels as occurs with hyperpolarization, the intracellular potassium concentration should be low, making the potassium equilibrium potential to be around zero. After washing the ACh out, the membrane gradually hyperpolarized. This change in the membrane potential might be due to diffusion of the high- $\mathrm{KCl}$ pipette solution into the patched cell, as well as into surrounding cells through gap junctions. The second application of ACh after a $10 \mathrm{~min}$ interval induced depolarization once again. After that, the membrane depolarized again, while the third application induced a brief hyperpolarization followed by a depolarization and the fourth application an even larger hyperpolarization. During this period of time, the endothelial cells seemed to recover their high intracellular potassium concentration. Similar responses were observed in 7 other preparations (3 luminal-surface-up and 4 abluminal-surface-up preparations).

As isolated endothelial cells seemed to loose their high intracellular potassium concentration, we attempted to reload potassium into the cells by incubating the preparation in a high-potassium solution. Figure 4 shows a representative result obtained from one of the 8 luminal-surface-up preparations used. The average resting membrane potential of the endothelial cells in these preparations was $-10.1 \pm 2.0 \mathrm{mV}$. Soon after establishment of the whole-cell clamp configuration, ACh induced a depolarization. After washing the ACh out, the potassium concentration in the bath solution was increased. The membrane potential jumped to a positive value and then gradually decreased to a steady level. After 20 minutes, the normal bath solution was reintroduced, but the membrane potential was still around zero (the average of 8 experiments, $-1.0 \pm 0.6 \mathrm{mV}$ ). Nevertheless, $\mathrm{ACh}$ induced a quick and large hyperpolarization followed by a slow depolarization, indicating that the intracellular potassium had recovered its high concentration. One might expect that in normal bath solution, the intracellular potassium would be depleted once again and that hyperpolarizations induced by 


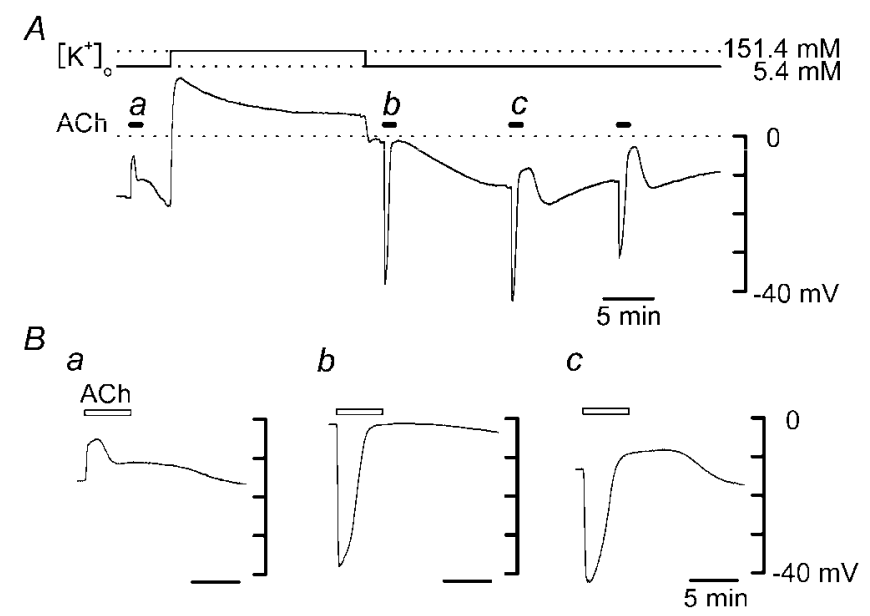

Fig. 4. The effect of incubation in high potassium solution on the ACh-induced voltage response in a luminal-surface-up mesenteric artery preparation. $A$ : The upper trace represents the external potassium concentration and the lower trace the membrane potential of an endothelial cell within the isolated endothelial layer. ACh $(3 \mu \mathrm{M})$ was applied during the periods indicated by the four bars. The small negative voltage deflections prior to the ACh-responses were artefacts on switching the bath solution. $B$ : ACh-induced voltage responses in expanded time scale. $a-c$ correspond to $a-c$ in $A$. ACh was applied during the periods indicated by the open boxes. Scales given in $c$ also apply to $a$ and $b$.

further application of ACh would decrease. However, by the third and the fourth application of ACh, large hyperpolarizations persisted, probably because a high concentration of potassium was supplied through the patch pipette as shown in Fig. 3.

\section{Discussion}

In the present experiments, we showed that endothelial cells were dependent on smooth muscle cells for both their membrane potential and their intracellular ionic composition. Once the endothelial layer was separated from the underlying smooth muscle layer, the endothelial cells could not maintain their high intracellular potassium concentration and their membrane potential.

Myoendothelial gap junctions pass electrical current which is carried by small ions (Sandow and Hill, 2000; Yamamoto et al., 2001). In the present experiments, when the smooth muscle and the endothelial layers remained in close apposition, the membrane potentials of both the smooth muscle and endothelial cells were closely correlated, with the endothelial cells slightly less polarized than the smooth muscle cells. This indicates that there is a continuous net current flowing from the endothelial cells to the smooth muscle cells. As action potentials of smooth muscle cells have been shown to be conducted into endothelial cells with a relative amplitude of $92.4 \%$ in guinea-pig mesenteric arterioles (Yamamoto et al., 2001), the current flowing through the myoendothelial gap junctions could maintain the membrane potential of the endothelial cells close to that of the smooth muscle cells even if the original membrane potential of the endothelia cells was nearly zero. 
The resting membrane potential was actually nearly zero in most of the isolated endothelial layers studied in the present experiments. Although several preparations showed relatively more negative membrane potentials of down to $-20 \mathrm{mV}$, the reason for these outliers was not investigated further. Vasoactive substances such as ACh, substance $\mathrm{P}$ and bradykinin induce membrane hyperpolarization in endothelial cells (Busse et al., 1988; Sakai, 1990) and this effect may be due to activation of an apamin-sensitive small-conductance $\mathrm{Ca}^{2+}$-activated $\mathrm{K}^{+}$channel and/ or a charybdotoxin-sensitive intermediate-conductance $\mathrm{Ca}^{2+}$-activated $\mathrm{K}^{+}$channel (Hashitani and Suzuki, 1997; Nishiyama et al., 1998; Yamamoto et al., 1999; Burnham et al., 2002; Bychkov et al., 2002). In the present experiments, ACh induced quick depolarization of the membrane in isolated endothelial layers. If this depolarization were induced by activation of the same $\mathrm{K}^{+}$channels as occurs for hyperpolarization, the intracellular concentration of potassium should be low making the $\mathrm{K}^{+}$equilibrium potential to be close to zero. Indeed, when the intracellular potassium concentration recovered to a high level either by intracellular dialysis with the pipette solution or by incubation in a high-potassium bath solution, ACh induced a membrane hyperpolarization. Although conductances other than that due to potassium might also be involved in the AChinduced depolarization, further investigation was not undertaken in the present experiments.

The resting membrane potential of the endothelial cells in isolated endothelial layers did not seem to be determined by potassium channels, as the membrane was still depolarized following restoration of the high intracellular concentration of potassium by incubation in a highpotassium bath solution. Instead nonselective cation channels having a reversal potential of close to zero, which have found in endothelial cells from various kinds of vessels (for review, see Nilius et al., 1997), might be responsible for the resting membrane potential. In the process of internal dialysis with pipette solution, the membrane gradually hyperpolarized first and then depolarized again, a typical example of which is shown in Fig. 3. The intracellular ionic composition should change dynamically during this period, which might affect the ionic mechanisms determining the resting membrane potential, although this was not investigated further in the present experiments. As the resting membrane potential of cultured endothelial cells is $\left[\mathrm{K}^{+}\right]_{0}$-dependent (Adams et al., 1989; Campbell et al., 1991), some potassium channels may be expressed during the culture process.

It is very likely that the intracellular ionic composition of endothelial cells is largely dependent on that of the underlying smooth muscle cells, because the intracellular capacity of the endothelium is much smaller compare to that of the smooth muscle layer. Due to the strong influences provided by the smooth muscle cells via myoendothelial gap junctions, expression of the $\mathrm{Na}^{+}-\mathrm{K}^{+}$pump seems to be downregulated in endothelial cells of mesenteric arteries. Expression of the $\mathrm{Na}^{+}-\mathrm{K}^{+}$pump has been detected in cultured endothelial cells that are not influenced by smooth muscle cells (Daut et al., 1988; Oike et al., 1993; Zahler et al., 1996; Orlov et al., 2004).

Resting membrane potentials have been reported in freshly isolated endothelial cells (Daut et al., 1988; Katnik and Adams, 1995; von Beckerath et al., 1996) and in cultured endothelial cells (Olesen et al., 1988; Sharma and Davis, 1994; Vaca et al., 1996; Voets et al., 1996), with values that are generally more negative than those in the present experiments. In the rat aorta, the resting membrane potential and the ACh response are not much different between isolated 
endothelial cells and endothelial cells in intact vascular segment (Marchenko and Sage, 1993, 1994). Membrane potential measurements in isolated cells are routinely performed using either conventional or perforated whole-cell clamp methods, in which intracellular dialysis with pipette solution occurs quickly. Thus, the true membrane potential of an isolated single cell under original conditions cannot be measured using this method.

In the present experiments, on the other hand, we did not use isolated single cells but isolated endothelial layers. Within an isolated endothelial layer, the individual endothelial cells are electrically coupled to each other making a syncytium. As the intercellular electrical resistance between endothelial cells is quite low (Yamamoto et al., 2001), the entire syncytium should be isopotential under the static conditions. Once the conventional whole-clamp configuration has been established, the patched cell is rapidly dialyzed with pipette solution, which has a high- $\mathrm{KCl}$ concentration. The pipette solution then diffuses gradually into the surrounding cells through the gap junctions, resulting in the syncytium no longer being isopotential under these dynamic conditions. The membrane potential is now the weighted average of the membrane potentials of the individual cells within the syncytium, and the closer the cell is to the patched cell, the larger the contribution is. The process is not too fast and the change in membrane potential during this process can be monitored (see Fig. 3). Thus, the measured membrane potential immediately after the establishment of the whole-cell clamp condition is most likely to be the original membrane potential in the syncytium.

In conclusion, vascular smooth muscle cells and endothelial cells are influencing each other as a functional unit. Endothelial cells produce vasoactive substances such as nitric oxide and prostacyclin which control smooth muscle contractility. Smooth muscle cells, on the other hand, maintain both the intracellular ionic composition and the resting membrane potential of endothelial cells. From this resting membrane potential, the endothelial cells produce active hyperpolarization in response to ACh or other agonists, which again affects smooth muscle contractility (the so-called EDHF phenomenon).

\section{References}

Adams, D.J., Barakeh, J., Laskey, R. and van Breemen, C. (1989). Ion channels and regulation of intracellular calcium in vascular endothelial cells. FASEB J. 3: 2389-2400.

Alvarez-Maubecin, V., García-Hernández, F., Williams, J.T. and Van Bockstaele, E.J. (2000). Functional coupling between neurons and glia. J. Neurosci. 20: 4091-4098.

Burnham, M.P., Bychkov, R., Félétou, M., Richards, G.R., Vanhoutte, P.M., Weston, A.H. and Edwards, G. (2002). Characterization of an apamin-sensitive small-conductance $\mathrm{Ca}^{2+}$-activated $\mathrm{K}^{+}$channel in porcine coronary artery. Br. J. Pharmacol. 135: 1133-1143.

Busse, R., Fichtner, H., Lückhoff, A. and Kohlhardt, M. (1988). Hyperpolarization and increased free calcium in acetylcholine-stimulated endothelial cells. Am. J. Physiol. 255: H965-H969.

Bychkov, R., Burnham, M.P., Richards, G.R., Edwards, G., Weston, A.H., Félétou, M. and Vanhoutte, P.M. (2002). Characterization of a charybdotoxin-sensitive intermediate conductance $\mathrm{Ca}^{2+}$ activated $\mathrm{K}^{+}$channel in porcine coronary endothelium: relevance to EDHF. Br. J. Pharmacol. 137: 1346-1354.

Campbell, D.L., Strauss, H.C. and Whorton, A.R. (1991). Voltage-dependence of bovine pulmonary artery endothelial cell function. J. Mol. Cell Cardiol. 23 (Suppl. 1): 133-144. 
Daut, J., Mehrke, G., Nees, S. and Newman, W.H. (1988). Passive electrical properties and electrogenic sodium transport of cultured guinea-pig coronary endothelial cells. J. Physiol. (Lond.) 402: 237254.

Hashitani, H. and Suzuki, H. (1997). $\mathrm{K}^{+}$channels which contribute to the acetylcholine-induced hyperpolarization in smooth muscle of the guinea-pig submucosal arteriole. J. Physiol. (Lond.) 501: 319-329.

Katnik, C. and Adams, D.J. (1995). An ATP-sensitive potassium conductance in rabbit arterial endothelial cells. J. Physiol. (Lond.) 485: 595-606.

Marchenko, S. and Sage, S.O. (1993). Electrical properties of resting and acetylcholine-stimulated endothelium in intact rat aorta. J. Physiol. (Lond.) 462: 735-751.

Marchenko, S. and Sage, S.O. (1994). Mechanism of acetylcholine action on membrane potential of endothelium of intact rat aorta. Am. J. Physiol. 266: H2388-H2395.

Nilius, B., Viana, F. and Droogmans, G. (1997). Ion channels in vascular endothelium. Annu. Rev. Physiol. 59: 145-170.

Nishiyama, M., Hashitani, H., Fukuta, H., Yamamoto, Y. and Suzuki, H. (1998). Potassium channels activated in the endothelium-dependent hyperpolarization in guinea-pig coronary artery. $J$. Physiol. (Lond.) 510: 455-465.

Oike, M., Droogmans, G., Casteels, R. and Nilius, B. (1993). Electrogenic $\mathrm{Na}^{+} / \mathrm{K}^{+}$-transport in human endothelial cells. Pflügers Arch. 424: 301-307.

Olesen, S.-P., Davies, P.F. and Clapham, D.E. (1988). Muscarinic-activated $\mathrm{K}^{+}$current in bovine aortic endothelial cells. Circ. Res. 62: 1059-1064.

Orlov, S.N., Thorin-Trescases, N., Pchejetski, D., Taurin, S., Farhat, N., Tremblay, J., Thorin, E. and Hamet, P. (2004). $\mathrm{Na}^{+} / \mathrm{K}^{+}$pump and endothelial cell survival: $\left[\mathrm{Na}^{+}\right]_{\mathrm{i}} /\left[\mathrm{K}^{+}\right]_{\mathrm{i}}$-independent necrosis triggered by ouabain, and protection against apoptosis mediated by elevation of $\left[\mathrm{Na}^{+}\right]_{\mathrm{i}}$. Pflügers Arch. 448: 335-345.

Sakai, T. (1990). Acetylcholine induces Ca-dependent K currents in rabbit endothelial cells. Jpn. J. Pharmacol. 53: 235-246.

Sandow, S.L. and Hill, C.E. (2000). Incidence of myoendothelial gap junctions in the proximal and distal mesenteric arteries of the rat is suggestive of a role in endothelium derived hyperpolarizing factor-mediated responses. Circ. Res. 86: 341-346.

Sharma, N.R. and Davis, M.J. (1994). Mechanism of substance P-induced hyperpolarization of porcine coronary artery endothelial cells. Am. J. Physiol. 266: H156-H164.

Vaca, L., Licea, A. and Possani, L.D. (1996). Modulation of cell membrane potential in cultured vascular endothelium. Am. J. Physiol. 270: C819-C824.

Voets, T., Droogmans, G. and Nilius, B. (1996). Membrane currents and the resting membrane potential in cultured bovine pulmonary artery endothelial cells. J. Physiol. (Lond.) 497: 95-107.

von Beckerath, N., Dittrich, M., Klieber, H.-G. and Daut, J. (1996). Inwardly rectifying $\mathrm{K}^{+}$channels in freshly dissociated coronary endothelial cells from guinea-pig heart. J. Physiol. (Lond.) 491: 357-365.

Yamamoto, Y., Imaeda, K. and Suzuki, H. (1999). Endothelium-dependent hyperpolarization and intercellular electrical coupling in guinea-pig mesenteric arterioles. J. Physiol. (Lond.) 514: 505513.

Yamamoto, Y., Klemm, M.F., Edwards, F.R. and Suzuki, H. (2001). Intercellular electrical communication among smooth muscle and endothelial cells in guinea-pig mesenteric arterioles. J. Physiol. (Lond.) 535: 181-195.

Zahler, R., Sun, W., Ardito, T. and Kashgarian, M. (1996). Na-K-ATPase $\alpha$-isoform expression in heart and vascular endothelia: cellular and developmental regulation. Am. J. Physiol. 270: C361-C371. 